\title{
SOME PROPERTIES OF JOST FUNCTIONS FOR SCHRÖDINGER EQUATION WITH DISTRIBUTION POTENTIAL
}

\author{
R.CH. KULAEV, A.B. SHABAT
}

\begin{abstract}
The work is devoted to the substantial extension of the space of the potentials in the inverse scattering problem for the linear Schrödinger equation on the real axis. We consider the Schrödinger operator with a potential in the space of generalized functions. This extension includes not only the potential like delta function, but also exotic cases like Cantor functions. In this way we establish the conditions on existence and uniqueness of Jost solutions. We study their analytic properties. We provide some estimates for the Jost solutions and their derivatives. We show that the Schrödinger equation with the distribution potential can be uniformly approximated by the equations with smooth potentials.
\end{abstract}

Keywords: inverse scattering problem, Schrödinger equation, Jost functions, delta-type potential, singular potential, distribution potential.

Mathematics Subjects Classifications: 34L25, 35J10, 37K15

This paper is devoted to extending the class of the potentials in the inverse scattering problem for the linear Schrödinger equation on the real axis

$$
\psi_{x x}=\left(q(x)+k^{2}\right) \psi, \quad x \in \mathbb{R} .
$$

While studying the inverse scattering problem, a fundamental role is played by the analytic properties of a special fundamental system of solutions to the Schrödginer equation with an exponentially growing asymptotic at infinity (Jost solutions). In classical scattering theory equation (1) is considered under the condition that the potential $q(x)$ is a summable function satisfying the condition

$$
\int_{-\infty}^{\infty}(1+|x|)|q(x)| d x<\infty .
$$

The conditions for the function $q$ arises naturally from the existence and uniqueness of the Jost solution as well as from the possibility of their analytic continuation for the complex values of the parameter $k[1]-[3]$.

In the present work we study the mathematical properties of Schrödinger equation (1) with the potentials being generalized derivatives of the functions of bounded variation. An interest to the equations with generalized coefficients increases constantly these days (see, for instance, [4] - 10] and the references therein). Study of Schrödinger equation (1) with the potentials being generalized derivatives of the functions of bounded variation extends the class of problem, which can be analyzed, but a pure mathematical feature of this object is to be taken into consideration.

R.Ch. Kulaev, A.B. Shabat, Some properties of Jost functions for Schrödinger equation With Distribution POTENTIAL.

(C)Kulaev R.Ch., Shabat A.B. 2017.

The work is supported by Russian Science Foundation (Grant no. 15-11-20007).

Submitted May 23, $201 \%$. 
In what follows we study what conditions the function $q$ should satisfy to ensure not only the existence and uniqueness of Jost solution, but also the possibility of their analytic continuation from the imaginary axis in the complex plane $k$. These issues are studied in Section 1 of the present paper. We also prove the continuity of the Jost solutions on the entire real axis and we establish basic estimates. In the second section we study more gentle properties of the Jost solutions, we show that their solutions belong to $W_{\text {loc }}^{1,1}$ and we prove the estimates for their derivatives. In the third section we show that the Schrödinger operator with a potential being a generalized derivative of a function of a bounded variation can be regarded as the uniform limit of the operators with smooth potentials. As it follows from the results of works [4], 5], in this case all solutions of the differential equation belong to $W_{\text {loc }}^{1,1}$.

1. Jost solutions. Let $Q$ be a real function defined on the entire real axis $\mathbb{R}$ and

$$
V_{Q}(x)=\sup \sum_{i=1}^{m}\left|Q\left(x_{i+1}\right)-Q\left(x_{i}\right)\right|
$$

where the supremum is taken over all $m \in \mathbb{N}$ and all $\left\{x_{i}\right\}_{i=1}^{m}$ such that

$$
-\infty<x_{1}<x_{2}<\ldots<x_{m} \leqslant x<\infty
$$

We define the total variation of the function $Q$ as the limit $V_{Q}=\lim _{x \rightarrow \infty} V_{Q}(x)$. By $B V$ we denote the space of all real function having a bounded variation on $\mathbb{R}$, while $\mathcal{M}$ stands for the set of all functions, each being a generalized derivative of some function in $B V$.

We consider Schrödinger equation (1), in which the potential $q$ is an element of $\mathcal{M}$ and $q=Q^{\prime}, Q \in B V$. First of all we are interested in existence of the Jost solution for the considered equation. As we have mentioned above, the Jost solution form a fundamental system of solutions depending on the parameter $k$ with exponential asymptotics on the negative and positive infinities. The standard method employed the theory of ordinary differential equations for proving the unique solvability of equations subject to initial condition consists in passing to an equivalent integral equation. In this case the method of successive approximations allows one to obtain the existence conditions for the equation with the initial data. Since equation (1) has many solutions with a prescribed asymptotics at infinity, the passage to the limit as $x_{0} \rightarrow \infty$ in the Cauchy problem with the initial data at a finite point $x_{0} \in \mathbb{R}$ does not solve the issue. This is why in order to construct the fundamental system of solutions, one has to use separately the conditions as $x \rightarrow \infty$ and as $x \rightarrow-\infty$. Moreover, there is also the problem on the analytic continuation of the fundamental system of solutions as functions of the complex parameter $k$. Exactly the analyticity condition distinguishes the Jost solutions.

Let $K_{+}=\{k \in \mathbb{C}$, $\operatorname{Re} k \geqslant 0\}$. By $\psi^{ \pm}(x, k), x \in \mathbb{R}, k \in K_{+}$, we denote the functions introduced by the relations

$$
\begin{aligned}
& \psi^{+}(x, k)=\phi^{+}(x, k) e^{k x}, \quad \phi^{+}(x, k)=1+\int_{-\infty}^{x} R(x-y, k) \phi^{+}(y, k) d Q(y), \\
& \psi^{-}(x, k)=\phi^{-}(x, k) e^{-k x}, \quad \phi^{-}(x, k)=1+\int_{x}^{\infty} R(y-x, k) \phi^{-}(y, k) d Q(y),
\end{aligned}
$$

in which

$$
R(y, k)=\frac{1-e^{-2 k y}}{2 k} \theta(y)
$$


and $\theta(y)$ is the Heaviside function. At that, the integrals in (2) are treated in the RiemannStieltjes sense and kernel (3) satisfies the estimate

$$
|R(y, k)| \leqslant \int_{-y}^{0}\left|e^{2 k s}\right| d s \leqslant y, \quad y \geqslant 0, \quad \operatorname{Re} k \geqslant 0
$$

in $K_{+}$.

The functions $\psi^{ \pm}$introduced by means of relations (2) are called Jost solutions to Schrödinger equation. These special solutions satisfy the conditions $\psi^{+}(x, k) \sim e^{k x}$ as $x \rightarrow-\infty$ and $\psi^{-}(x, k) \sim e^{-k x}$ as $x \rightarrow \infty$.

Theorem 1. For each $k \in K_{+}$, the Jost solutions $\psi^{ \pm}(x, k)$ of Schrödinger equation (1) are determined uniquely by relations (2) provided the condition holds:

$$
\int_{-\infty}^{\infty}(1+|x|) d V_{Q}(x)<\infty .
$$

For each fixed $k \in K_{+}$, the solutions $\phi^{ \pm}(x, k)$ to integral equations (2) are continuous on the entire real axis, and for each fixed $x \in \mathbb{R}$ they are continuous functions of the parameter $k$ in $K_{+}$. Moreover, $\phi^{ \pm}(x, k)$ are analytic in the half-plane $\operatorname{Re} k>0$ and satisfies the following estimates:

(i)

$$
\begin{aligned}
& \left|\phi^{+}(x, k)-1\right| \leqslant \exp \left\{\int_{-\infty}^{x} V_{Q}(y) d y\right\} \int_{-\infty}^{x} V_{Q}(y) d y \\
& \left|\phi^{-}(x, k)-1\right| \leqslant \exp \left\{\int_{x}^{\infty}\left(V_{Q}-V_{Q}(y)\right) d y\right\} \int_{x}^{\infty}\left(V_{Q}-V_{Q}(y)\right) d y, \quad k \in K_{+} ;
\end{aligned}
$$

(ii)

$$
\begin{aligned}
& \left|\phi^{+}(x, k)-1\right| \leqslant C^{+}(1+\max \{x, 0\}) \int_{-\infty}^{x}(1+|x|) d V_{Q}(x), \\
& \left|\phi^{-}(x, k)-1\right| \leqslant C^{-}(1+\max \{-x, 0\}) \int_{x}^{\infty}(1+|x|) d V_{Q}(x),
\end{aligned}
$$

where the constants $C^{ \pm}$are independent of $k \in K_{+}$;

(iii)

$$
\begin{array}{r}
\left|\phi^{+}(x, k)-1\right| \leqslant \frac{V_{Q}(x)}{|k|} \exp \left\{\frac{V_{Q}(x)}{|k|}\right\} \leqslant \frac{V_{Q}}{|k|} \exp \left\{\frac{V_{Q}}{|k|}\right\}, \\
\left|\phi^{-}(x, k)-1\right| \leqslant \frac{V_{Q}-V_{Q}(x)}{|k|} \exp \left\{\frac{V_{Q}-V_{Q}(x)}{|k|}\right\} \leqslant \frac{V_{Q}}{|k|} \exp \left\{\frac{V_{Q}}{|k|}\right\},
\end{array}
$$

where $k \in K_{+} \backslash\{0\}$.

Proof. We employ the method of successive approximations to find the conditions under which there exist the Jost solutions to problem (2). In view of the analogy, we make the calculations 
only for the first equation. Let us show that as $k \in K_{+}$, the solution $\phi^{+}(x, k)$ of the associated integral equation exists and can be found as

$$
\phi^{+}(x, k)=1+\sum_{n=1}^{\infty} f_{n}(x, k), \quad f_{n+1}(x, k)=\int_{-\infty}^{x} R(x-y, k) f_{n}(y, k) d Q(y), \quad f_{0}(x, k)=1 .
$$

In order to prove the convergence of series (6), we employ inequality (4):

$$
\left|f_{1}(x, k)\right| \leqslant \int_{-\infty}^{x}|R(x-y, k)| d V_{Q}(y) \leqslant \int_{-\infty}^{x}(x-y) d V_{Q}(y)=\left.(x-y) V_{Q}(y)\right|_{-\infty} ^{x}+\int_{-\infty}^{x} V_{Q}(y) d y .
$$

Letting

$$
\lim _{x \rightarrow-\infty}|x| V_{Q}(x)=0,
$$

we obtain the estimate for $\left|f_{1}(x, k)\right|$

$$
\left|f_{1}(x, k)\right| \leqslant \int_{-\infty}^{x}(x-y) d V_{Q}(y)=\int_{-\infty}^{x} V_{Q}(y) d y=M(x)
$$

Let us show that the terms of series (6) admit the estimate

$$
\left|f_{n}(x, k)\right| \leqslant \frac{M^{n}(x)}{n !} .
$$

By induction we get

$$
\left|f_{n+1}(x, k)\right| \leqslant \int_{-\infty}^{x}|R(x-y, k)|\left|f_{n}(y, k)\right| d V_{Q}(y)=\frac{1}{n !} \int_{-\infty}^{x}(x-y) M^{n}(y) d V_{Q}(y) .
$$

Integrating by parts, we finally obtain

$$
\begin{aligned}
\left|f_{n+1}(x, k)\right| & \leqslant \frac{1}{n !} \int_{-\infty}^{x} V_{Q}(y) M^{n}(y) d y-\frac{1}{(n-1) !} \int_{-\infty}^{x} V_{Q}^{2}(y)(x-y) M^{n-1}(y) d y \\
& \leqslant \frac{1}{n !} \int_{-\infty}^{x} V_{Q}(y) M^{n}(y) d y=\frac{M^{n+1}(x)}{(n+1) !}
\end{aligned}
$$

It follows from estimate (8) that series of perturbation theory (6) is majorized by the series

$$
e^{M(x)}=1+\sum_{n=1}^{\infty} \frac{M^{n}(x)}{n !} .
$$

This is why we can state that provided the condition

$$
\int_{-\infty}^{0}|x| d V_{Q}(x)<\infty
$$

holds, series (6) converges uniformly on each segment $(-\infty, b], b<\infty$. Indeed, inequality (5) ensures that (7) holds and $M(b)<\infty$. This implies that the function $\phi^{+}(x, k)$ is continuous as $\operatorname{Re} k \geqslant 0$, is analytic as $\operatorname{Re} k>0$ and satisfies the corresponding inequality in (i). 
In the same way we can obtain that under the condition

$$
\int_{0}^{\infty} x d V_{Q}(x)<\infty
$$

the function $\phi^{-}(x, k)$ is continuous as $\operatorname{Re} k \geqslant 0$ and is analytic as $\operatorname{Re} k>0$ and satisfies the corresponding inequality in (i).

Combining conditions $(9)$ and $(10)$ into one condition, we obtain the sufficient condition for solvability of integral equations (2)

$$
\int_{-\infty}^{\infty}|x| d V_{Q}(x) \leqslant \int_{-\infty}^{\infty}(1+|x|) d V_{Q}(x)<\infty .
$$

We proceed to proving inequality (ii) for $\phi^{+}(x, k)$. Employing (4), we get

$$
\begin{aligned}
\left|\phi^{+}(x, k)\right| & \leqslant 1+\int_{-\infty}^{x}(x-y)\left|\phi^{+}(y, k)\right| d V_{Q}(y) \\
& \leqslant 1+\int_{-\infty}^{0}|y|\left|\phi^{+}(y, k)\right| d V_{Q}(y)+\int_{-\infty}^{x} x\left|\phi^{+}(y, k)\right| d V_{Q}(y) .
\end{aligned}
$$

Since $\left|\phi^{+}(y, k)\right| \leqslant e^{M(0)}$ as $y \leqslant 0$, it follows from (5) that

$$
\left|\phi^{+}(x, k)\right| \leqslant C+\int_{-\infty}^{x} x\left|\phi^{+}(y, k)\right| d V_{Q}(y), \quad 1<C<\infty
$$

and the constant $C$ is independent of $k \in K_{+}$. We divide both sides of the integral inequality by $C(1+|x|)$ and introduce the notation $\chi(x, k)=\frac{\phi^{+}(x, k)}{C(1+|x|)}$ to obtain the inequality

$$
|\chi(x, k)| \leqslant 1+\int_{-\infty}^{x}(1+|y|)|\chi(y, k)| d V_{Q}(y)
$$

which in view of (5) can be solved by the iteration method:

$$
\begin{aligned}
& \left|\chi_{0}(x, k)\right|=1 \\
& \left|\chi_{1}(x, k)\right| \leqslant \int_{-\infty}^{x}(1+|y|) d V_{Q}(y)=(1+x) V_{Q}(x)-\int_{-\infty}^{x} V_{Q}(y) d|y|=M_{1}(x), \\
& d M_{1}(x)=(1+x) d V_{Q}(x)+V_{Q}(x) d x-V_{Q}(x) d x=(1+x) d V_{Q}(x), \\
& \left|\chi_{n+1}(x, k)\right| \leqslant \int_{-\infty}^{x}(1+|y|) \frac{M_{1}^{n}(y)}{n !} d V_{Q}(y)=\frac{1}{n !} \int_{-\infty}^{x} M_{1}^{n}(y) d M_{1}(y)=\frac{M_{1}^{n+1}(x)}{(n+1) !} .
\end{aligned}
$$


Therefore, $\left|\phi^{+}(x, k)\right| \leqslant C_{1}(1+|x|) e^{M_{1}(x)}$. Hence, by (11) and (i) for $x \geqslant 0$ we get

$$
\begin{aligned}
\left|\phi^{+}(x, k)-1\right| \leqslant & \int_{-\infty}^{0}|y|\left|\phi^{+}(y, k)\right| d V_{Q}(y)+x \int_{-\infty}^{x}\left|\phi^{+}(y, k)\right| d V_{Q}(y) \\
& \leqslant C_{2}(1+x) \int_{-\infty}^{x}(1+|y|) d V_{Q}(y)
\end{aligned}
$$

In view of (i) for negative $x$ we get

$$
\left|\phi^{+}(x, k)-1\right| \leqslant e^{M(0)} \int_{-\infty}^{x}(1+|y|) d V_{Q}(y) .
$$

Comparing the latter inequality with (13), we get estimate (ii) for $\phi^{+}$.

The proof of (iii) follows the same lines with the only difference that instead of estimate (4) one should employ the obvious inequality $|R(y, k)| \leqslant \frac{1}{|k|}, k \in K_{+} \backslash\{0\}$. Indeed, by the latter estimate we get the integral inequality

$$
\left|\phi^{+}(x, k)-1\right| \leqslant \frac{V_{Q}(x)}{|k|}+\frac{1}{|k|} \int_{-\infty}^{x}\left|\phi^{+}(y, k)-1\right| d V_{Q}(y),
$$

which can be solved easily by the iteration method:

$$
\begin{aligned}
& \left|g_{0}(x, k)\right| \leqslant \frac{V_{Q}(x)}{|k|}, \quad\left|g_{1}(x, k)\right| \leqslant \frac{1}{|k|} \int_{-\infty}^{x} \frac{V_{Q}(x)}{|k|} d V_{Q}(y)=\frac{V_{Q}^{2}(x)}{2|k|^{2}} \\
& \left|g_{n}(x, k)\right| \leqslant \frac{1}{n !|k|} \int_{-\infty}^{x} \frac{V_{Q}^{n}(x)}{|k|^{n}} d V_{Q}(y)=\frac{V_{Q}^{n+1}(x)}{(n+1) !|k|^{n+1}} .
\end{aligned}
$$

It remains to prove that for each fixed $k \in K_{+}$the function $\phi^{+}(x, k)$ is continuous on the entire real axis. Taking into consideration the uniform convergence of series (6) on the half-axis $(-\infty, a)$ for each $a \in \mathbb{R}$, it is sufficient to prove the continuity of the iterations $f_{n}(x, k)$ w.r.t. $x$. Integrating by parts, we get:1

$$
\begin{aligned}
f_{0}(x, k)=1 & \\
f_{n+1}(x, k)= & \int_{-\infty}^{x} e^{-2 k(x-y)} f_{n}(y, k) Q(y) d y-\int_{-\infty}^{x} \frac{1-e^{-2 k(x-y)}}{2 k} f_{n}^{\prime}(y, k) Q(y) d y, \\
f_{n+1}^{\prime}(x, k)= & f_{n}(x, k) Q(x)-2 k \int_{-\infty}^{x} e^{-2 k(x-y)} f_{n}(y, k) Q(y) d y \\
& -\int_{-\infty}^{x} e^{-2 k(x-y)} f_{n}^{\prime}(y, k) Q(y) d y .
\end{aligned}
$$

\footnotetext{
${ }^{1}$ Hereinafter to denote the derivative of the function $f(x, k)$ w.r.t. the variable $x$ we employ one of the following notations: $f^{\prime}, f_{x}$ or $\frac{\partial f}{\partial x}$. In each case the choice of the notations is determined by the presence of a subscript or a superscript in the notation for the function.
} 
Condition (5) implies that $\int_{-\infty}^{a} V_{Q}(x) d x<\infty$ for each $a \in \mathbb{R}$. And since the inequality $|Q(x)|<$ $V_{Q}(x)$ holds everywhere on $\mathbb{R}$, condition (5) ensures that $Q \in L^{1}(-\infty, a)$ for each $a \in \mathbb{R}$. Now the continuity of the function $f_{n}(x, k)$ in the variable $x$ is obvious.

The arguing for the function $\phi^{-}(x, k)$ is similar. The proof is complete.

Corollary 1. If the potential $q$ is compactly supported, the Jost solutions are analytic in the entire complex plane $k$.

Corollary 2. As $|k| \rightarrow \infty$, Re $k \geqslant 0$, the following limits hold true:

$$
\begin{aligned}
& \phi^{+}(x, k)=1+\int_{-\infty}^{x} R(x-y, k) d Q(y)+o\left(\frac{1}{k}\right), \\
& \phi^{-}(x, k)=1+\int_{x}^{\infty} R(y-x, k) d Q(y)+o\left(\frac{1}{k}\right),
\end{aligned}
$$

Proof. It follows from (2) and statement (iii) that

$$
\phi^{ \pm}(x, k)=1+O\left(\frac{1}{k}\right) \quad \text { as } \quad|k| \rightarrow \infty, \quad \operatorname{Re} k \geqslant 0 .
$$

Substituting these relations into (2), we obtain (15).

Remark 1. Replacing the parameter $k \mapsto-k$, we arrive at one more pair of the functions $\varphi^{\mp}$ satisfying the conditions $\varphi^{+}(x, k) \sim e^{k x}$ as $x \rightarrow \infty$ and $\varphi^{-}(x, k) \sim e^{-k x}$ as $x \rightarrow-\infty$. It is obvious that $\varphi^{\mp}(x, k)=\psi^{ \pm}(x,-k)$. Moreover, for each $x \in \mathbb{R}$ the solutions $\varphi^{\mp}(x, k)$ are continuous in $k$ as $\operatorname{Re} k \leqslant 0$ and analytic in $k$ as $\operatorname{Re} k<0$.

2. Further properties of the Jost solutions. Proven Theorem 1 allows us to obtain the properties of the solutions $\phi^{ \pm}(x, k)$ to integral equations (2) as well as the properties of the Jost solutions to Schrödinger equation (1).

In the space $B V$ we choose a subspace of the functions satisfying condition (5) and we introduce the notation $B V_{1}$ for this subspace. We shall assume that the real potential $q$ of the equation (1) is a generalized derivative of some function $Q \in B V_{1}$. Since $q=Q^{\prime}$ in the generalized sense, the function $Q$ is recovered by the potential $q$ up to an additive constant. This is why we have a certain freedom in choosing this constant. While studying the properties of the Jost solution $\psi^{+}$, it will be convenient for us to assume that $Q(x) \rightarrow 0$ as $x \rightarrow-\infty !^{1}$ In particular, if the potential $q$ is compactly supported and $\operatorname{supp} q \subset[a, b]$, we let $Q(x)=0$ as $x \leqslant a$. In the same way, while studying the properties of the other solution $\psi^{-}$we assume $Q(x) \rightarrow 0$ as $x \rightarrow \infty$.

We consider integral equations (2) and let us show that their solutions $\phi^{ \pm}$belong to $W_{\text {loc }}^{1,1}$ and moreover, $\phi_{x}^{ \pm} \in B V_{\text {loc }}$. Since the arguing is similar, we consider only the solution $\phi^{+}$. It is obvious that it is sufficient to prove that $\phi^{+} \in W^{1,1}(a, b), \phi_{x}^{+} \in B V(a, b)$ for an arbitrary finite interval $(a, b) \subset \mathbb{R}$. According (6),

$$
\phi^{+}(x, k)=\sum_{n=0}^{\infty} f_{n}(x, k),
$$

where the functions $f_{n}$ satisfies identities (14). It follows from Theorem 1 that as $k \in K_{+}$, the series converges at each point $x \in \mathbb{R}$. We denote by $\phi_{n}$ the $n$th particular sum of series (6).

\footnotetext{
${ }^{1}$ In this case the potential $q$ is a finite Borel measure on $\mathbb{R}$.
} 
For for a fixed $k \in K_{+}$we obtain

$$
\begin{aligned}
& \phi_{0}(x, k)=1, \quad \phi_{1}(x, k)=1+\int_{-\infty}^{x} e^{-2 k(x-y)} Q(y) d y, \\
& \phi_{1}^{\prime}(x, k)=Q(x)-2 k \int_{-\infty}^{x} e^{-2 k(x-y)} Q(y) d y=\int_{-\infty}^{x} e^{-2 k(x-y)} d Q(y) .
\end{aligned}
$$

By $B V(a, b) \subset L^{1}(a, b)$ this implies the belongings $\phi_{1}(x, k) \in W^{1,1}(a, b), \phi_{1}^{\prime}(x, k) \in B V(a, b)$. Since $Q \in B V$, at each point $x \in \mathbb{R}$ the function $Q$ has one-sided limits $Q(x \pm 0)$. At that, the set of discontinuity points of the function $Q$ is at most countable. This is why at the continuity points of $Q$, the function $\phi_{1}$ possesses the derivative $\phi_{1}^{\prime}$ w.r.t. $x$. If at some point $x_{0} \in \mathbb{R}$ the function $Q$ has a jump $\triangle Q\left(x_{0}\right)=Q\left(x_{0}+0\right)-Q\left(x_{0}-0\right)$, then the derivative $\phi_{1}^{\prime}$ has the same by size jump. Therefore, we can extend the function $\phi_{1}^{\prime}(x, k)-Q(x)$ by the continuity on the entire segment $[a, b]$ and assume that $\phi_{1}^{\prime}(x, k)-Q(x) \in A C[a, b]$.

By (14), for $n \geqslant 1$ we have

$$
\begin{aligned}
\phi_{n+1}(x, k)= & 1+\int_{-\infty}^{x} e^{-2 k(x-y)} \phi_{n}(y, k) Q(y) d y-\int_{-\infty}^{x} \frac{1-e^{-2 k(x-y)}}{2 k} \phi_{n}^{\prime}(y, k) Q(y) d y, \\
\phi_{n+1}^{\prime}(x, k)= & \phi_{n}(x, k) Q(x)-2 k \int_{-\infty}^{x} e^{-2 k(x-y)} \phi_{n}(y, k) Q(y) d y \\
& -\int_{-\infty}^{x} e^{-2 k(x-y)} \phi_{n}^{\prime}(y, k) Q(y) d y .
\end{aligned}
$$

Arguing by induction, we get

$$
\phi_{n}(x, k) \in W^{1,1}(a, b), \quad \phi_{n}^{\prime}(x, k) \in B V(a, b), \quad \phi_{n}^{\prime}(x, k)-Q(x) \phi_{n-1}(x, k) \in A C[a, b]
$$

for all $n \in \mathbb{N}$. Moreover, the left hand sides in (16) can be rewritten as

$$
\begin{aligned}
\phi_{n+1}(x, k)= & +\int_{-\infty}^{x} e^{-2 k(x-y)} \phi_{n}(y, k) Q(y) d y \\
& -\int_{-\infty}^{x} R(x-y, k)\left(\int_{-\infty}^{y} e^{-2 k(y-s)} \phi_{n-1}(s, k) d Q(s)\right) Q(y) d y \\
\phi_{n+1}^{\prime}(x, k)= & \int_{-\infty}^{x} e^{-2 k(x-y)} \phi_{n}(y, k) d Q(y) .
\end{aligned}
$$

In view of (5), (8), this implies easily the estimate

$$
\left|\phi_{n+1}^{\prime}(x, k)\right| \leqslant \int_{-\infty}^{x}\left|e^{-2 k(x-y)}\right|\left|\phi_{n}(y, k)\right| d V_{Q}(y) \leqslant V_{Q} \sum_{m=0}^{n} \frac{\left(\int_{-\infty}^{x} V_{Q}(y) d y\right)^{m}}{m !} .
$$

Therefore, the sequence $\phi_{n}^{\prime}-Q \phi_{n-1}$ converges uniformly on $[a, b]$. Due to the uniform on $[a, b]$ convergence

$$
\phi_{n} \rightrightarrows \phi^{+}, \quad \phi_{n}^{\prime}-Q \phi_{n-1} \rightrightarrows \phi_{x}^{+}-Q \phi^{+}
$$


and relations 17 we obtain

$$
\phi^{+}(x, k) \in W^{1,1}(a, b), \quad \phi_{x}^{+}(x, k) \in B V(a, b), \quad \phi_{x}^{+}(x, k)-Q(x) \phi^{+}(x, k) \in A C[a, b] .
$$

Thus, we have proved the following theorem.

Theorem 2. For each fixed $k \in K_{+}$, the belonging $\phi^{ \pm} \in W_{\mathrm{loc}}^{1,1}$ holds. Moreover, $\phi_{x}^{ \pm} \in B V_{\mathrm{loc}}$ and the difference $\phi_{x}^{ \pm}-Q \phi^{ \pm}$is absolutely continuous on finite segments in $\mathbb{R}$.

It follows from Theorem 2 and (18), (19) that

$$
\phi_{x}^{+}(x, k)=\int_{-\infty}^{x} e^{-2 k(x-y)} \phi^{+}(y, k) d Q(y), \quad \phi_{x}^{-}(x, k)=-\int_{x}^{\infty} e^{-2 k(y-x)} \phi^{-}(y, k) d Q(y),
$$

and the estimates

$$
\begin{aligned}
&\left|\phi_{x}^{+}(x, k)\right| \leqslant \int_{-\infty}^{x}\left|e^{-2 k(x-y)}\right|\left|\phi^{+}(y, k)\right| d V_{Q}(y), \\
&\left|\phi_{x}^{-}(x, k)\right| \leqslant \int_{x}^{\infty}\left|e^{-2 k(x-y)}\right|\left|\phi^{-}(y, k)\right| d V_{Q}(y) .
\end{aligned}
$$

hold true.

Substituting estimate (ii) of Theorem 1 into formulae (21), we obtain the following statements.

Lemma 1. For each $k \in K_{+}$, the derivatives $\phi_{x}^{ \pm}(x, k)$ are uniformly bounded

$$
\begin{aligned}
& \left|\phi_{x}^{+}(x, k)\right| \leqslant C \int_{-\infty}^{x}(1+|y|) d V_{Q}(y) \leqslant C \int_{-\infty}^{\infty}(1+|y|) d V_{Q}(y), \\
& \left|\phi_{x}^{-}(x, k)\right| \leqslant C \int_{x}^{\infty}(1+|y|) d V_{Q}(y) \leqslant C \int_{-\infty}^{\infty}(1+|y|) d V_{Q}(y) .
\end{aligned}
$$

Lemma 2. As $|k| \rightarrow \infty$, Re $k \geqslant 0$, the limiting relations $\phi_{x}^{ \pm}(x, k) \rightarrow 0$ hold.

In the same way, by 21 and $(12)$ we get the next statement.

Lemma 3. For each $k \in K_{+}$, the derivatives $\phi_{x}^{ \pm}(x, k)$ satisfy the estimates

$$
\begin{array}{ll}
\left|\phi_{x}^{+}(x, k)\right| \leqslant C_{1} V_{Q}(x), & x \leqslant 0, \\
\left|\phi_{x}^{-}(x, k)\right| \leqslant C_{2} V_{Q}(x), & x \geqslant 0,
\end{array}
$$

where the constants $C_{1}, C_{2}$ are independent of $k$.

Lemma 4. If $\operatorname{Re} k>0$, then $\phi_{x}^{+}(x, k) \rightarrow 0$ as $x \rightarrow \infty$ and $\phi_{x}^{-}(x, k) \rightarrow 0$ as $x \rightarrow-\infty$.

Proof. By (21) we have

$$
\left|\phi_{x}^{+}(x, k)\right| \leqslant\left|e^{-2 k x}\right| \int_{-\infty}^{x}\left|e^{2 k y}\right|\left|\phi^{+}(y, k)\right| d V_{Q}(y) .
$$

In view of (5) and estimate (iii) of Theorem 1, the right hand side of the inequality tends to zero as $x \rightarrow \infty$ only if $\operatorname{Re} k>0$.

The properties of the functions $\phi^{ \pm}$and of their derivatives allow us to specify Theorem 2 . 
Lemma 5. If $-\infty<a<\infty$, then for each $k \in K_{+} \backslash\{0\}$ we have $\phi^{+}-1 \in W^{1,1}(-\infty, a)$ and $\phi^{-}-1 \in W^{1,1}(a, \infty)$.

Indeed, the belonging $\phi^{+} \in L^{1}(-\infty, a)$ follows estimate (iii) of Theorem 1 and (5), while the belonging $\phi_{x}^{+} \in L^{1}(-\infty, a)$ is implied by Lemma 3 and again by condition (5).

We note that as $k=0$, Lemma 5 is not true as the next example shows.

Example 1. We consider equation (1) with the potential $q(x)=x^{-\frac{8}{3}}$ as $x \geqslant 1$. It is easy to confirm that condition (5) is satisfied. Moreover, for $x \geqslant 1$ we have

$$
\psi^{-}(x, 0)=\phi^{-}(x, 0)=\frac{x^{\frac{2}{3}}}{3}\left[\operatorname{ch}\left(3 x^{-\frac{1}{3}}\right)-\frac{x^{\frac{1}{3}}}{3} \operatorname{sh}\left(3 x^{-\frac{1}{3}}\right)\right] .
$$

Employing Taylor formula, we get the asymptotic identity

$$
\phi^{-}(x, 0)=1+\frac{9}{10} x^{-\frac{2}{3}}+o\left(x^{-\frac{2}{3}}\right) \text { as } x \rightarrow \infty,
$$

which implies obviously that $\phi^{-}-1 \notin L^{1}(a, \infty)$ for each $a \in \mathbb{R}$.

3. Approximation by smooth potentials. In this section we show that in the inverse scattering problem, the Schrödinger equation with the potential $q \in \mathcal{M}, q=Q^{\prime}, q \in B V_{1}$ can be uniformly approximated by the equations with smooth potentials. We note that a similar result for an equation on a finite interval was obtained in work [5] (see also [4]).

We still assume that $q=Q^{\prime}$ in the generalized sense and $Q \in B V_{1}$. Let $\eta_{\varepsilon}(x)$ be the standard averaging kernel. For a function $Q \in B V_{1}$ we define the mean function $Q_{\varepsilon}=\eta_{\varepsilon} * Q$ on $\mathbb{R}$. Then for each partition $-\infty<x_{0}<x_{1}<\ldots<x_{n} \leqslant x<\infty$ we have

$$
\begin{aligned}
\sum_{i=1}^{n}\left|Q_{\varepsilon}\left(x_{i}\right)-Q_{\varepsilon}\left(x_{i-1}\right)\right| & \leqslant \int_{-\infty}^{\infty} \eta_{\varepsilon}(y) \sum_{i=1}^{n}\left|Q\left(x_{i}-y\right)-Q\left(x_{i-1}-y\right)\right| d y \\
& \leqslant \int_{-\varepsilon}^{\varepsilon} \eta_{\varepsilon}(y) V_{Q}(x-y) d y \leqslant V_{Q}(x+\varepsilon) .
\end{aligned}
$$

Therefore, $V_{Q_{\varepsilon}}(x) \leqslant V_{Q}(x+\varepsilon) \leqslant V_{Q}$ and

$$
\int_{-\infty}^{0}|x| d V_{Q_{\varepsilon}}(x)=\int_{-\infty}^{0} V_{Q_{\varepsilon}}(x) d x-\left.x V_{Q_{\varepsilon}}(x)\right|_{-\infty} ^{0} \leqslant \int_{-\infty}^{0} V_{Q_{\varepsilon}}(x) d x \leqslant \int_{-\infty}^{0} V_{Q}(x+\varepsilon) d x<\infty .
$$

In the same one can show that

$$
\int_{0}^{\infty}|x| d V_{Q_{\varepsilon}}(x)<\infty
$$

The obtained inequalities imply in particular that $Q_{\varepsilon} \in B V_{1}$.

Remark 2. It is easy to see that for each $x, y \in \mathbb{R}, x>y$, the inequality

$$
\left|Q_{\varepsilon}(x)-Q_{\varepsilon}(y)\right| \leqslant V_{Q_{\varepsilon}}(x) \leqslant V_{Q}(x+\varepsilon)
$$

holds true. As $y \rightarrow-\infty$, we obtain

$$
\left|Q_{\varepsilon}(x)\right| \leqslant V_{Q_{\varepsilon}}(x) \leqslant V_{Q}(x+\varepsilon), \quad x \in \mathbb{R} .
$$


Therefore, $Q_{\varepsilon} \in L^{1}(-\infty, b)$ for each $b \in \mathbb{R}$. Moreover, Lebesgue theorem implies that

$$
\int_{-\infty}^{x}\left|Q_{\varepsilon}(y)-Q(y)\right| d y \rightarrow 0
$$

for each $x \in \mathbb{R}$.

By $\phi_{\varepsilon}^{+}$we denote the solution to the integral equation

$$
\phi_{\varepsilon}^{+}(x, k)=1+\int_{-\infty}^{x} R(x-y, k) \phi_{\varepsilon}^{+}(x, k) d Q_{\varepsilon}(y) .
$$

Since $Q_{\varepsilon} \in B V_{1}$, the solution $\phi_{\varepsilon}^{+}$exists for each $k \in K_{+}$. By $\psi_{\varepsilon}^{+}=e^{k x} \phi_{\varepsilon}^{+}$we denote the Jost solution to the Schrödginer equation equation with the smooth potential $q_{\varepsilon}=Q_{\varepsilon}^{\prime}$.

Lemma 6. For each $k \in K_{+}$, as $\varepsilon \rightarrow 0$, the limits hold true:

(1) $\psi_{\varepsilon}^{+}(x, k) \rightarrow \psi^{+}(x, k)$ for each $x \in \mathbb{R}$;

(2) $\frac{\partial}{\partial x} \psi_{\varepsilon}^{+}(x, k) \rightarrow \frac{\partial}{\partial x} \psi^{+}(x, k)$ almost everywhere in $\mathbb{R}$.

Proof. Since the function $Q$ is continuous almost everywhere in $\mathbb{R}$ and the Jost solutions are continuous on the entire axis $\mathbb{R}$, it is sufficient to prove the statement of the lemma at the continuity points of the function $Q$.

Let $x \in \mathbb{R}$ and $k \in K_{+}$be arbitrary and fixed and the function $Q$ is continuous at the point $x$. Then by Theorem 1 and $(20)$, for each $\varepsilon \in[0,1]$ the identities

$$
\phi_{\varepsilon}^{+}(x, k)=\lim _{n \rightarrow \infty} \phi_{\varepsilon, n}(x, k), \quad \frac{\partial}{\partial x} \phi_{\varepsilon}^{+}(x, k)=\lim _{n \rightarrow \infty} \phi_{\varepsilon, n}^{\prime}(x, k),
$$

hold, where (see (14)) $\phi_{\varepsilon, 0}(x, k)=1$,

$$
\begin{aligned}
\phi_{\varepsilon, n+1}(x, k)= & \int_{-\infty}^{x} e^{-2 k(x-y)} \phi_{\varepsilon, n}(y, k) Q_{\varepsilon}(y) d y-\int_{-\infty}^{x} R(x-y, k) \phi_{\varepsilon, n}^{\prime}(y, k) Q_{\varepsilon}(y) d y \\
\phi_{\varepsilon, n+1}^{\prime}(x, k)= & \phi_{\varepsilon, n}(x, k) Q_{\varepsilon}(x)-2 k \int_{-\infty}^{x} e^{-2 k(x-y)} \phi_{\varepsilon, n}(y, k) Q_{\varepsilon}(y) d y \\
& -\int_{-\infty}^{x} e^{-2 k(x-y)} \phi_{\varepsilon, n}^{\prime}(y, k) Q_{\varepsilon}(y) d y
\end{aligned}
$$

Here we assume that the value $\varepsilon=0$ is associated with the function $\phi^{+}=\phi_{0}^{+}$. It follows from inequalities (8), (19), (22) that both limits in (23) are uniform in $\varepsilon \in[0,1]$. Hence, it is obvious that to prove the lemma, it is sufficient to show that as $\varepsilon \rightarrow 0$, for each $n$ the relations $\phi_{\varepsilon, n}^{(j)}(x, k) \rightarrow \phi_{n}^{(j)}(x, k), j=0,1$, hold true.

As $n=0$, the limiting relations are obvious. Arguing by induction, we assume that $\phi_{\varepsilon, n}^{(j)}(x, k) \rightarrow \phi_{n}^{(j)}(x, k)$ for all $n \leqslant N$ and let us show that $\phi_{\varepsilon, N+1}^{(j)}(x, k) \rightarrow \phi_{N+1}^{(j)}(x, k)$ as $\varepsilon \rightarrow 0$. But these relations are implied by inequalities (8), (22) ensuring the possibility to pass to the limit in the integral in expressions (24). Indeed, by (8), (19) and (22), as $y \in(-\infty, x]$, we get 
the estimates

$$
\begin{aligned}
& \left|e^{-2 k(x-y)} \phi_{\varepsilon, n}(y, k) Q_{\varepsilon}(y)\right| \leqslant e^{M_{\varepsilon}(y)}\left|Q_{\varepsilon}(y)\right| \leqslant C e^{M(y)}|Q(y)| \in L_{1}(-\infty, x), \\
& \left|\phi_{\varepsilon, n}^{\prime}(y, k)\right| \leqslant e^{M_{\varepsilon}(y)} V_{Q_{\varepsilon}}(y) \leqslant e^{M(y+\varepsilon)} V_{Q}(y+\varepsilon), \quad M_{\varepsilon}(y)=\int_{-\infty}^{y} V_{Q_{\varepsilon}}(s) d s, \\
& \left|R(x-y, k) \phi_{\varepsilon, n}^{\prime}(y, k) Q_{\varepsilon}(y)\right| \leqslant(x-y) e^{M_{\varepsilon}(y)} V_{Q_{\varepsilon}}(y)\left|Q_{\varepsilon}(y)\right| \leqslant(x-y) e^{M(y+\varepsilon)} V_{Q}(y+\varepsilon)|Q(y)| .
\end{aligned}
$$

And since

$$
\int_{-\infty}^{x}(x-y) e^{M(y)} V_{Q}(y)|Q(y)| d y \leqslant \int_{-\infty}^{x}(x-y) V_{Q}^{2}(y) d y \leqslant x \int_{-\infty}^{x} V_{Q}^{2}(y) d y-\int_{-\infty}^{x} V_{Q}^{2}(y) y d y
$$

by $\lim _{y \rightarrow-\infty} y V_{Q}(y)=0$, the right hand of the inequality does not exceed

$$
x \int_{-\infty}^{x} V_{Q}^{2}(y) d y+\int_{-\infty}^{x} y^{2} V_{Q}(y) d V_{Q}(y)<\infty .
$$

This allows us to apply the Lebesgue theorem on majorized convergence in (24) as $n=N$. The proof is complete.

Theorem 3. Let $k \in K_{+}$. Then for each $b \in \mathbb{R}$, the uniform limiting relation $\psi_{\varepsilon}^{+} \rightrightarrows \psi^{+}$ holds on the half-line $(-\infty, b]$ as $\varepsilon \rightarrow 0$, while on the half-line $[b, \infty)$ we have $\psi_{\varepsilon}^{-} \rightrightarrows \psi^{-}$.

Proof. Let $b \in \mathbb{R}$ and $k \in K_{+}$be fixed. By Theorem 1 and inequality (22), for an arbitrary $\tau>0$ there exists $a \in(-\infty, 0)$ independent of $\varepsilon$ such that

$$
\left|\psi_{\varepsilon}^{+}(x, k)-\psi^{+}(x, k)\right| \leqslant\left|\phi_{\varepsilon}^{+}(x, k)-\phi^{+}(x, k)\right|<\frac{\tau}{3}, \quad x \in(-\infty, a] .
$$

If $a \geqslant b$, the theorem is proved.

Let $a<b$. We denote by $\psi_{\varepsilon}^{*}$ the solution to differential equation (1) with the smooth potential $q_{\varepsilon}$ subject to the initial conditions

$$
\psi_{\varepsilon}^{*}(a, k)=\psi^{+}(a, k), \quad \frac{\partial}{\partial x} \psi_{\varepsilon}^{*}(a, k)=\frac{\partial}{\partial x} \psi^{+}(a+0, k) .
$$

Then for each $x \in[a, b]$ we have

$$
\left|\psi_{\varepsilon}^{+}(x, k)-\psi^{+}(x, k)\right| \leqslant\left|\psi_{\varepsilon}^{+}(x, k)-\psi_{\varepsilon}^{*}(x, k)\right|+\left|\psi_{\varepsilon}^{*}(x, k)-\psi^{+}(x, k)\right| .
$$

Let us show that for sufficiently small $\varepsilon$ both absolute values in the right hand side are less than $\frac{\tau}{3}$ for all $x \in[a, b]$. The functions $\psi_{\varepsilon}^{+}$and $\psi_{\varepsilon}^{*}$ are solutions to the same differential equation with the smooth potential $q_{\varepsilon}$. By Lemma 5, as $\varepsilon \rightarrow 0$ we have

$$
\psi_{\varepsilon}^{+}(a, k) \rightarrow \psi^{+}(a, k), \quad \frac{\partial}{\partial x} \psi_{\varepsilon}^{+}(a, k) \rightarrow \frac{\partial}{\partial x} \psi^{+}(a+0, k) .
$$

Hence, thanks to the continuous dependence on the initial data for the solutions to the Schrödginer equation with a smooth potential (see, for instance, [11, Ch. 2, Sect. 4]), for sufficiently small $\varepsilon$, the inequality

$$
\left|\psi_{\varepsilon}^{+}(x, k)-\psi_{\varepsilon}^{*}(x, k)\right|<\frac{\tau}{3}
$$

holds for all $x \in[a, b]$. Concerning the difference $\left|\psi_{\varepsilon}^{*}(x, k)-\phi^{+}(x, k)\right|$, by result of work [5] mentioned in the beginning of this section, this difference tends to zero as $\varepsilon \rightarrow 0$ uniformly in $[a, b]$. 
Therefore, for sufficiently small $\varepsilon$, the inequality

$$
\left|\psi_{\varepsilon}^{+}(x, k)-\psi^{+}(x, k)\right|<\tau, \quad x \in(-\infty, b],
$$

holds true. This completes the proof for the solution $\psi^{+}$. The arguing for $\psi^{-}$is similar.

\section{BIBLIOGRAPHY}

1. L.D. Faddeev. The inverse problem in the quantum theory of scattering // Uspekhi Matem. Nauk. 14:4(88), 57-119 (1959). [J. Math. Phys. 4:1, 72-104 (1963).]

2. P. Deift, E. Trubowitz. Inverse scattering on the line // Comm. Pure Appl. Math. 32:2, 121-251 (1979).

3. V.A. Marchenko. Sturm-Liouville operators and their applications. Naukova Dumka, Kiev (1977). (in Russian).

4. A.M. Savchuk, A.A. Shkalikov. Inverse problems for Sturm-Liouville operators with potentials in Sobolev spaces: uniform stability // Funkts. Anal. Pril. 44:4, 34-53 (2010). [Funct. Anal. Appl. 44:4, 270-285 (2010).]

5. A.M. Savchuk, A.A. Shkalikov. Sturm-Liouville operators with singular potentials // Matem. Zamet. 66:6, 897-912 (1999). [Math. Notes. 66:6, 741-753 (1999).]

6. R.O. Hryniv, Ya.V. Mykytyuk. Eigenvalue asymptotics for Sturm-Liouville operators with singular potentials // J. Funct. Anal. 238:1, 27-57 (2006).

7. M.Sh. Badakhov, A.B. Shabat. Darboux transformations in the inverse scattering problem // Ufimskij Matem. Zhurn. 8:4, 43-52 (2016). [Ufa Math J. 8:4, 42-51 (2016).]

8. A.B. Shabat. Inverse spectral problem for delta potentials // Pisma ZhETF. 102:9, 705-708 (2015). [JETP Lett. 102:9, 620-623 (2015).]

9. A.B. Shabat. Difference Schrödinger equation and quasisymmetric polynomials // Teor. Matem. Fiz. 184:2, 16-27 (2015). [Theor. Math. Phys. 184:2, 1067-1077 (2015).]

10. R.Ch. Kulaev, A.B. Shabat. Inverse scattering problem for compactly supported potentials in the space of Borel measures // Preprint SMI VSC RAS. 2, (2016).

11. E.A. Coddington, N. Levinson. Theory of ordinary differential equations. McGill-Hill Book Company, New York (1955).

Ruslan Chermenovich Kulaev,

North-Ossetia State Univeristy named after K.L. Khetagurov,

Vatutin str., 46,

362025, Vladikavkaz, Russian

South Mathematical Institute, VSC RAS,

Markus str., 22,

362027, Vladikavkaz, Russia

E-mail: kulaev@smath.ru

Alexey Borisovich Shabat,

L.D. Landau Institute for Theoretical Physics, RAS,

Academician Semenov av. 1-A, 142432, Chernogolovka, Russia,

E-mail: shabatab@mail.ru 